\title{
Effectiveness of intraoperative Magnesium Sulphate on acute pain management after major abdominal surgery
}

\author{
I. Cindea, V. Gherghina, A. Balcan, C. B. Samoila, S. State, A. Rosu \\ Anesthesia-Intensive Care Department, Emergency Clinical Hospital of Constanta, Romania \\ Faculty of General Medicine, Ovidius University of Constanta, Romania
}

\section{Goal of Study}

Although Magnesium Sulphate injected intraoperatively as an intravenous bolus has been reported to be of interest as adjuvant to early postoperative analgesia, the evidence is still inconsistent in this regard.

The objective of our prospective randomized, double-blind, placebo-controlled study is to evaluate the impact of a single intravenous dose of Magnesium Sulphate administered $1 \mathrm{~h}$ before the end of the procedure, on acute postoperative pain relief, during first $24 \mathrm{~h}$ after major abdominal surgery.

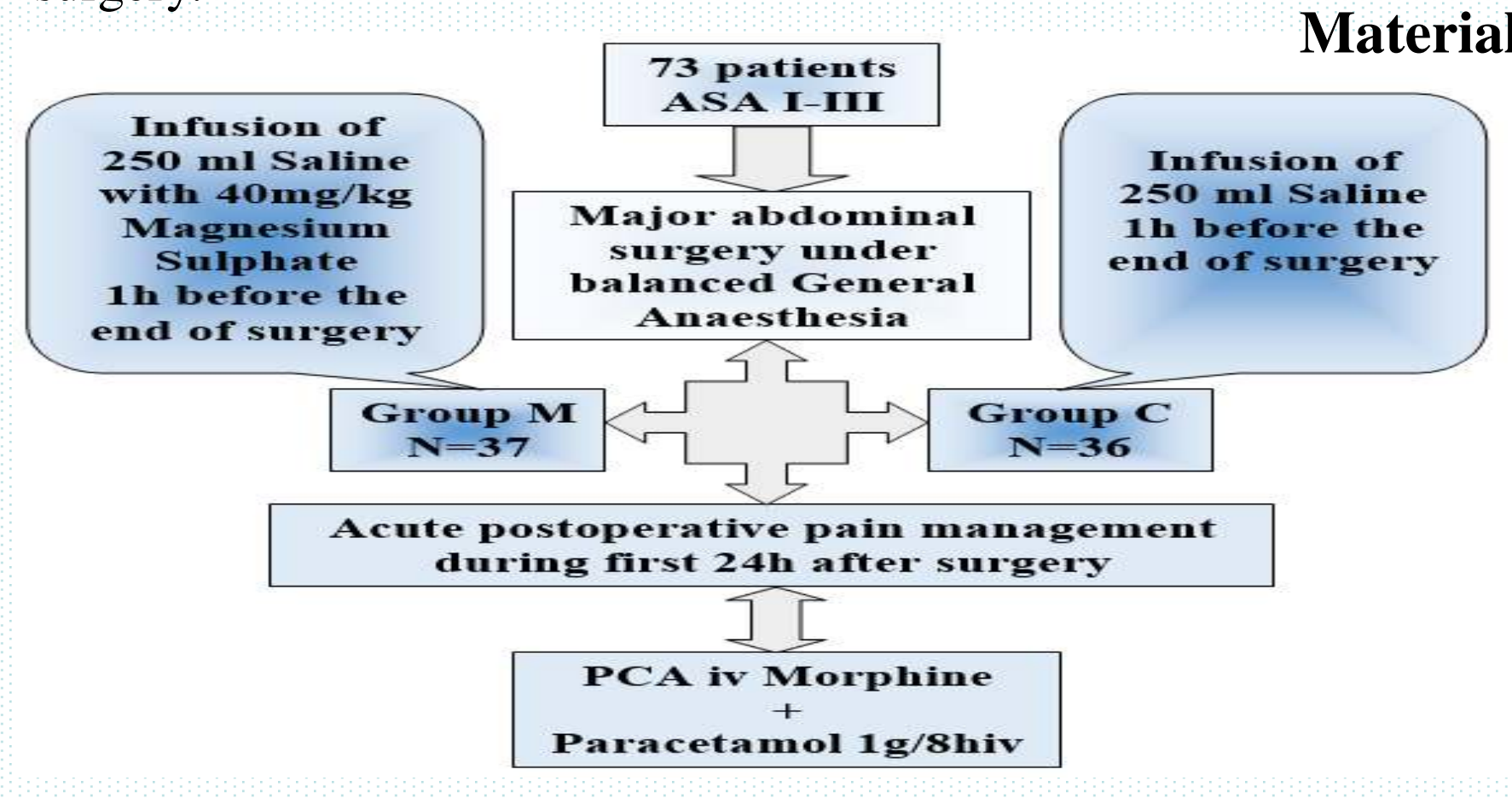
Primary end-points:
Intensity of acute postoperative pain at rest, assessed by VAS at $1 \mathrm{~h}$, $6 \mathrm{~h}, 12 \mathrm{~h}, 24 \mathrm{~h}$ after procedure; Intensity of acute postoperative pain while coughing, assessed by VAS at $1 \mathrm{~h}, 6 \mathrm{~h}, 12 \mathrm{~h}, 24 \mathrm{~h}$ after procedure; Total Morphine consumption during first $24 \mathrm{~h}$ after major abdominal surgery.
Secondary end-points:
Incidence of shivering; Incidence of nausea/vomiting; Incidence of hemodynamic disturbances; Evaluation of patient satisfaction using a subjective 4-level scale $(0$ - not satisfied at all; 1 - only slightly satisfied; 2 - somewhat satisfied; 3 - very satisfied).

\section{Results and Discussion}

VAS score trend by time revealed a significantly better management of postoperative pain at rest, as well as while coughing, during first $24 \mathrm{~h}$ after surgery, for group M compared to control. Our results are in line with literature reports on analgesia-potentiating effect of Magnesium sulphate, this benefit being even amplified by its preemptive administration, $1 \mathrm{~h}$ before the end of surgery.

\begin{tabular}{|c|c|c|c|c|}
\hline & \multicolumn{3}{|c|}{ VAS } \\
\hline Study Group & $\mathbf{1 h}$ & $\mathbf{6 h}$ & $\mathbf{1 2 h}$ & $\mathbf{2 4 h}$ \\
\hline $\begin{array}{c}\text { Group M } \\
\mathbf{N}=\mathbf{3 7}\end{array}$ & $1.1+/-0.5$ & $1.4+/-0.8$ & $2.2+/-0.7$ & $2.8+/-0.6$ \\
\hline $\begin{array}{c}\text { Group C } \\
\mathbf{N}=\mathbf{3 5}\end{array}$ & $2.4+/-0.6$ & $2.9+/-0.7$ & $3.5+/-0.9$ & $4.5+/-0.7$ \\
\hline p & $<0.05$ & $<0.05$ & $<0.05$ & $<0.05$ \\
\hline
\end{tabular}

\begin{tabular}{|c|c|c|c|c|}
\hline & \multicolumn{5}{|c|}{ VAS } \\
\hline Study Group & $\mathbf{1 h}$ & $\mathbf{6 h}$ & $\mathbf{1 2 h}$ & $\mathbf{2 4 h}$ \\
\hline $\begin{array}{c}\text { Group M } \\
\text { N=37 }\end{array}$ & $2.8+/-0.6$ & $3.2+/-0.4$ & $3.8+/-0.3$ & $4.5+/-0.2$ \\
\hline $\begin{array}{c}\text { Group C } \\
\text { N=36 }\end{array}$ & $4.6+/-0.9$ & $4.9+/-0.6$ & $5.3+/-0.8$ & $6.0+/-0.7$ \\
\hline p & $<0.05$ & $<0.05$ & $<0.05$ & $<0.05$ \\
\hline
\end{tabular}

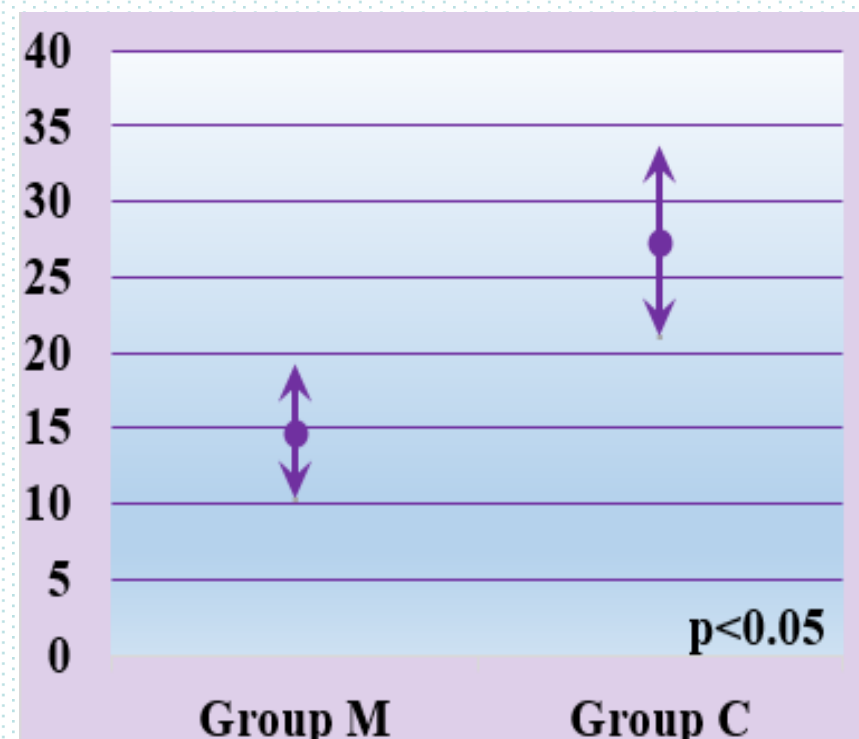

Figure 1 The evaluation of total Morphine requirement $(\mathrm{mg})$
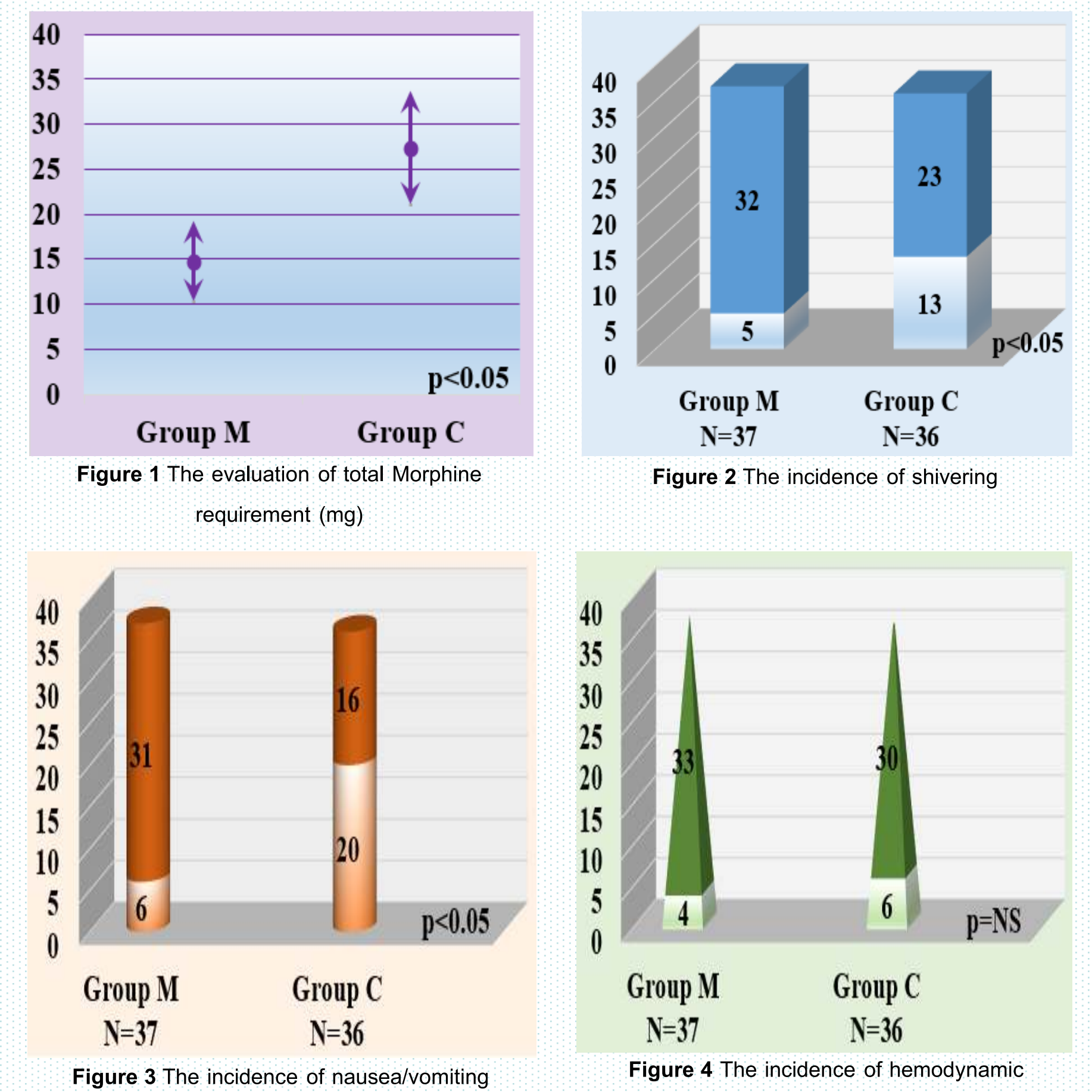

disturbances
A significant decrease in Morphine usage was noted in group M compared to group C. This reduction in opioid consumption is explained by antinociceptive effect of Magnesium Sulphate. Shivering and nausea/vomiting episodes registered during first $24 \mathrm{~h}$ postoperatively showed significantly lower rates in Magnesium-treated patients correlated with their decreased Morphine request.

We didn't document any deleterious cardiovascular impact of the analgesic therapy during study period. Besides, the parameters of hemodynamic profile were comparable in both groups.

Evaluation of patient satisfaction, another objective of our study, pointed out that most of the patients in group $\mathbf{M}$ rated their early postoperative status as being optimal, at least regarding the acute pain control, whereas in group $C$ the number of subjects fully satisfied with their analgesic regimen is significantly less representative. These results could be interpreted taking into account the analgesic efficacy of Magnesium Sulphate.

\begin{tabular}{|c|c|c|c|c|}
\hline & \multicolumn{3}{|c|}{ Patient satisfaction scale (0-3) } \\
\hline $\begin{array}{c}\text { Study group } \\
\text { Grade 0 }\end{array}$ & $\begin{array}{c}\text { Grade 1 (only } \\
\text { (not satisfied } \\
\text { at all) }\end{array}$ & $\begin{array}{c}\text { Grade } \\
\text { slightly } \\
\text { satisfied) }\end{array}$ & $\begin{array}{c}\text { Grade } \\
\text { (somewhat } \\
\text { satisfied) }\end{array}$ & $\begin{array}{c}\text { Grade 3 } \\
\text { (very } \\
\text { satisfied) }\end{array}$ \\
\hline $\begin{array}{c}\text { Group M } \\
\text { N=37 }\end{array}$ & $0 / 37$ & $0 / 37$ & $6 / 37$ & $31 / 37$ \\
\hline $\begin{array}{c}\text { Group C } \\
\text { N=36 }\end{array}$ & $4 / 36$ & $5 / 36$ & $11 / 36$ & $16 / 36$ \\
\hline p & $<0.05$ & $<0.05$ & $<0.05$ & $<0.05$ \\
\hline
\end{tabular}

\section{Conclusions}

Our results suggest that Magnesium Sulphate effectively attenuates acute postoperative pain, without serious adverse effects.

Thus, it should be recommended as an available and cheap adjuvant in multimodal analgesia protocol for managing the acute postoperative pain after major abdominal interventions. 